\title{
Prevalence of myopic macular degeneration worldwide: a systematic review and meta-analysis
}

\author{
Minjie Zou (1) , ${ }^{1,2}$ Shibin Wang, ${ }^{3}$ Aiming Chen, ${ }^{4}$ Zhenzhen Liu, ${ }^{1}$ \\ Charlotte Aimee Young, ${ }^{5}$ Yichi Zhang, ${ }^{6}$ Guangming Jin (ㄷ, , Danying Zheng ${ }^{1}$
}

\begin{abstract}
- Additional material is
published online only. To view please visit the journal online (http://dx.doi.org/10.1136/ bjophthalmol-2019-315298).

${ }^{1}$ State Key Laboratory of Ophthalmology, Zhongshan Ophthalmic Center, Sun Yat-Sen University, Guangzhou, China ${ }^{2}$ Department of Clinical Medicine, Zhongshan Schoo of Medicine, Sun Yat-sen University, Guangzhou, China ${ }^{3}$ Guangdong Mental Health Center, Guangdong General Hospital, Guangdong Academy of Medical Sciences, Guangzhou, China

${ }^{4}$ Department of Pharmacy, Fifth Affiliated Hospital of Sun Yatsen University, Zhuhai, China ${ }^{5}$ Department of Ophthalmology, University of California, San Francisco, California, USA ${ }^{6}$ Department of Ophthalmology, Guangdong Provincial Key Laboratory of Malignant Tumor Epigenetics and Gene Regulation, Sun Yat-sen University, Guangzhou, China
\end{abstract}

Correspondence to Dr Guangming Jin and Dr Danying Zheng, State Key Laboratory of Ophthalmology, Zhongshan Ophthalmic

Center, Sun Yat-sen University, Guangzhou 510060, China; guangming27050103@126. com, zhengdyy@163.com

$\mathrm{MZ}$ and SW are joint first authors.

Received 28 September 2019 Revised 10 February 2020 Accepted 14 February 2020 Published Online First 18 March 2020

\section{Check for updates}

(C) Author(s) (or their employer(s)) 2020. No commercial re-use. See rights and permissions. Published by BMJ.

To cite: Zou M, Wang $\mathrm{S}$, Chen $\mathrm{A}$, et al. Br J Ophthalmol 2020;104:1748-1754.
ABSTRACT
Purpose To investigate the prevalence of myopic macular degeneration (MMD) in the global population. Methods All published literature of population-based studies on MMD prevalence worldwide were searched and only those with clear definitions to diagnose and classify MMD lesions by standardised grading methods were selected. Meta-analysis methods were used to calculate the pooled prevalence of MMD and its $95 \% \mathrm{Cl}$ in a random-effects model. The prevalence of MMD lesions would also be reported, together with the subgroup analysis of age, region and gender. Correlation between MMD prevalence and spherical equivalent levels and axial length were also evaluated.

Results 12 studies with 58558 subjects were included in this meta-analysis. The pooled prevalence of MMD in the world population was $2.1 \%(95 \% \mathrm{Cl}$ : $1.3 \%$ to $3.3 \%$ ). In the subgroup analysis, people with the following characteristics were at higher risk of developing MMD: female, urban life, living in Asia, older age, longer axial length and severer myopia.

Conclusions MMD is a serious public health concern worldwide, particularly in subjects who are women, subjects living in urban areas, subjects living in Asia, and subjects with longer axial lengths and severer myopia. Further studies from other continents/ethnicities are needed for comprehensive estimates of the prevalence of MMD globally.

\section{INTRODUCTION}

Myopia has been one of the leading causes of visual impairment worldwide and the prevalence of myopia has been increasing in the past decades. Predictably, $49.8 \%$ of the world population will have myopia and 9.8\% will have high myopia by 2050 , which will be a great economic burden globally. Individuals with high myopia are at an increased potential risk of developing myopiarelated blinding complications, the most common of which is myopic macular degeneration (MMD), which could cause a progressive decrease in visual acuity. $^{1-5}$

MMD is one of the leading causes of blindness worldwide and usually causes a heavy economic burden to societies and individuals. ${ }^{36-11} \mathrm{MMD}$ has been reported as the first to the third most important cause of visual loss in Asian populations, indicating much more effort is needed for the guidance of future solutions. ${ }^{1}$

Evaluating the magnitude of MMD is of great importance for health authorities to understand its harmful impact and to develop appropriate preventive strategies. Though several studies performed in different countries reported the prevalence of $\mathrm{MMD},{ }^{6} 710$ 12-20 due to differences in diagnostic, definition and study locations, the prevalence of MMD in the general population varied significantly between studies, ranging from $0.2 \%$ to $10.7 \% .^{1519}$

Unfortunately, to the best of our knowledge, there has been no systematic review and metaanalysis in the prevalence of MMD published prior, which hinders people's understanding of the disease burden of MMD. Therefore, a better understanding of the magnitude of MMD is needed. We performed this systematic review and meta-analysis to address the prevalence of MMD in worldwide populations and performed subgroup analysis by evaluating different potential risk factors in worldwide populations.

\section{METHODS}

\section{Search strategy for literature}

This meta-analysis was conducted according to the Preferred Reporting Items for Systematic Reviews and Meta-Analyses statement. Publications reporting the prevalence of MMD among all populations worldwide were reviewed and assessed. Two investigators (GJ and $M Z$ ) searched the literature independently in both English (Embase, PubMed and Web of Science) and Chinese (SinoMed, WanFang and Chinese National Knowledge Infrastructure) databases until 19 July 2019. The search terms were as follows:

1. "Retinal Degeneration" $[$ Mesh]/[All fields) or "Macular Degeneration”[Mesh]/[All fields] or "Retinal Diseases"[Mesh]/[All fields] or "Retinal maculopathy"[All fields] or "Retinal complications"[All fields] or "Myopic macular degeneration"[All fields] or "Myopic maculopathy"[All fields].

2. "Refraction error" $[$ Mesh]/[All fields] or "Myopia”[Mesh]/[All fields] or "Myopia, Degenerative"[Mesh] or "Pathological myopia"[All fields] or "pathologic myopia"[All fields].

3. "Epidemiology" $[\mathrm{Mesh}] /[$ All fields $]$ or "Cohort Studies"[Mesh]/[All fields] or "Prevalence"[Mesh]/[All fields] or "Cross-Sectional Studies"[Mesh]/[All fields] or "Risk Factors" [Mesh]/[All fields]

4. Combine 1 AND 2 AND 3.

\section{Study selection}

Studies were included if they met the following criteria: (1) population-based study; (2) use 
recognised definitions and standardised grading method to diagnose and classify MMD lesions; (3) accessible full text in Chinese or English; (4) sample size $\geq 1000$; (5) response rate $\geq 60 \%$. $^{21} 22$ Several internationally recognised definitions and standardised grading methods are used for pathological myopia (PM) and PM Classification. ${ }^{7} 13141718$ Among the included studies, PM Classification developed by Vongphanit and the International Meta-Analyses of Pathological Myopia (META-PM) classification were the most used grading methods. According to PM Classification developed by Vongphanit, myopic retinopathy was graded into five categories including no myopic retinal lesion (category 0 ), tessellated fundus (category 1 ), diffuse chorioretinal atrophy (category 2), patchy chorioretinal atrophy (category 3 ) and macular atrophy (category 4). Four additional features to supplement these categories were defined as 'plus' lesions, namely lacquer cracks, myopic choroidal neovascularisation, Fuchs' spot and posterior staphyloma. Based on the International META-PM classification, the presence of MMD was defined and classified into the following categories: no macular lesions (category 0 ); tessellated fundus only (category 1 ); diffuse chorioretinal atrophy (category 2); patchy chorioretinal atrophy (category 3) and macular atrophy (category 4). 'Plus' lesions, which supplemented the Meta-PM categories, comprised lacquer cracks, choroidal neovascularisation (CNV) and Fuchs' spot. Based on fundus photograph grading, an eye was considered to have MMD if Meta-PM category 2, 3, 4 or any 'plus' lesion was observed. Studies using convenience sampling and those without details on the sampling method as well as studies focused on special populations (eg, patients with myopes) were also excluded.

Titles and abstracts of all initial searched results were screened independently by two investigators (GJ and MZ). If there was more than one publication based on the same study, the study with more complete information would be selected.

\section{Data extraction and quality assessment}

Two investigators (GJ and MZ) conducted the data extraction independently and any disagreements were resolved by a discussion with a third investigator (SW). The following information was extracted and tabulated: first author, study setting, sampling method, survey time, sample size, basic demographic data, and the prevalence of MMD and the specific lesions of pathological changes in MMD.

The quality of all selected articles was evaluated by two investigators (GJ and MZ) with a commonly used 8-item assessment tool. According to the quality evaluation tool, each study was given a score of $0-8$. We consider a score of $7-8$ as high quality, 4-6 as moderate quality and $0-3$ as low quality. The coding of assessment has been described previously. ${ }^{22-24}$

\section{Statistical analysis}

The meta-analysis was conducted using the Comprehensive Meta-Analysis software V.2 (Biostat, Englewood, New Jersey, USA). The prevalence of MMD and specific lesions of pathological changes in MMD with 95\% CIs were calculated using random-effects models. Heterogeneity between studies was assessed by $\mathrm{I}^{2}$ statistic, and $\mathrm{I}^{2}>50 \%$ was regarded as high heterogeneity. The age-specific pooled prevalence of MMD by $40-49$, 50-59, 60-69 and >70 years old age groups was conducted. To explore the possible sources of heterogeneity associated with gender, place of residence (rural/urban), area (Asia/NonAsia), survey year, refraction levels, grading systems and axial length levels, subgroup analyses were performed separately. In

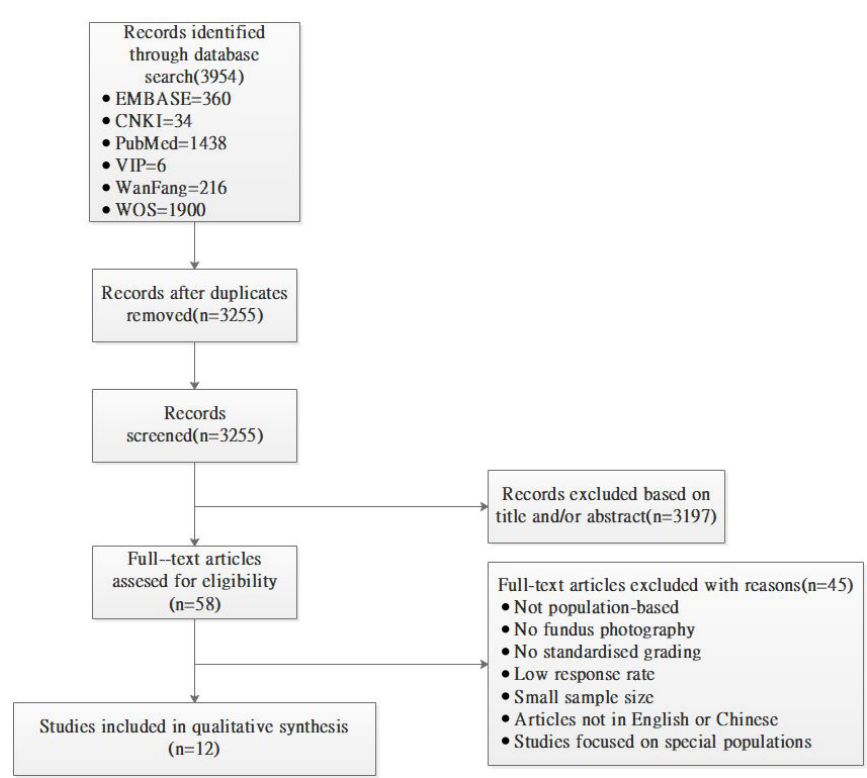

Figure 1 Flowchart of the study selection process.

the subgroup analysis of the grading scheme, we chose the two most commonly used grading systems (International META-PM Classification and PM Classification developed by Vongphanit). Continuous variables were dichotomised using median splitting method in subgroup analyses. According to the principle of meta-analysis, $\mathrm{I}^{2}>50 \%$ indicates great heterogeneity and in this situation, the random-effect model is suggested for conservativeness and reliability. Publication bias was assessed by the Funnel plots and Begg tests. The significance level was set at $\mathrm{p}<0.05$ (two-tailed). The funnel plot would be presented when the number of studies being meta-analysed is $>10$.

\section{RESULTS \\ Study selection and inclusion results with basic characteristics}

Figure 1 shows the selection process of studies identified through the database search with 3954 initial records. Altogether, 12 studies (13 datasets as one study provided two crosssectional with two different samples) ${ }^{20}$ with 58558 subjects were included for qualitative synthesis. The basic characteristics of included studies are given in table 1 . Of the 12 studies, 2 were in Chinese, ${ }^{12} 13$ and the remaining were in English. ${ }^{671014-2025} \mathrm{We}$ included 28356 individuals of five studies conducted in mainland China, ${ }^{612131618} 1058$ of one study in Taiwan China, ${ }^{14} 7702$ of two studies in Japan, ${ }^{17} 208716$ of one study in Singapore, ${ }^{7}$ 4582 of one study in the USA, ${ }^{15} 3583$ of one study in Australia, ${ }^{10}$ and 4561 of one study in India. ${ }^{19}$ All included studies have clear definitions of the target population and the samples are representative of the general population. Only one study graded subjects with myopia and the remaining 11 studies have all subjects graded for MMD. Some included studies report MMD prevalence among all subjects, ${ }^{13-15} 18-20$ whereas others also present MMD prevalence among high myopic subjects. ${ }^{6710121617}$ As for the grading in the included studies, seven studies had grading conducted by one investigator, ${ }^{6} 1214151819$ among which four studies had a second examination when necessary, ${ }^{6} 131519$ and the remaining studies had double grading. ${ }^{710161720}$ As several subgroup analyses (gender, age, spherical equivalent refraction levels and axial length levels) are not provided in all of the 
Table 1 Characteristics of the studies included in the meta-analysis

\begin{tabular}{|c|c|c|c|c|c|c|c|c|}
\hline Study (country/region) & Year examined & Area & Age range & $\mathrm{N}$ & $\begin{array}{l}\text { Male/Female } \\
\text { ratio }\end{array}$ & $\begin{array}{l}\text { Response } \\
\text { rate (\%) }\end{array}$ & $\begin{array}{l}\text { Grading } \\
\text { system }\end{array}$ & Assessment \\
\hline SEED (Singapore) & 2004-2011 & Urban & $\geq 40$ & 8716 & 0.98 & 75.6 & M & 7 \\
\hline The Shaanxi Eye Study (China) & 2003 & Rural & $1-91$ & 6815 & 0.87 & 80.2 & V & 6 \\
\hline The Yangxi Eye Study (China) & 2014 & Rural & $\geq 50$ & 4469 & 1.00 & 90.7 & $P$ & 6 \\
\hline The Shihpai Eye Study (Taiwan China) & 1999-2000 & Urban & $\geq 65$ & 1058 & 1.64 & 66.6 & A & 7 \\
\hline CHES (USA) & 2010-2013 & Urban & $\geq 50$ & 4582 & 0.58 & 79 & M & 6 \\
\hline The Handan Eye Study (China) & 2006-2007 & Rural & $\geq 30$ & 6603 & 0.87 & 90.4 & V & 6 \\
\hline The Beijing Eye Study (China) & 2001 & Urban \& Rural & $\geq 40$ & 4319 & - & 83.4 & V & 6 \\
\hline The Hisayama Eye Study (Japan) & 2005 & Urban & $\geq 40$ & 1892 & 0.70 & 42.7 & H & 5 \\
\hline The Blue Mountain Eye Study (Australia) & 1992-1994 & Urban & $\geq 49$ & 3583 & 1.30 & 82.4 & V & 8 \\
\hline The Central India Eye and Medical Study (India) & 2006 & Rural & $\geq 30$ & 4561 & 0.86 & 80.1 & $P$ & 7 \\
\hline The Hisayama Eye Study (Japan) & 2012 & Urban & $\geq 40$ & 2874 & 0.78 & 63.8 & M & 6 \\
\hline The Hisayama Eye Study (Japan) & 2017 & & & 2936 & 0.79 & 65.7 & & \\
\hline The Wuxi Eye Study (China) & 2010 & Urban & $\geq 50$ & 6150 & 0.74 & 90.7 & V & 6 \\
\hline
\end{tabular}

A, Avilla's Grading Method for PM; CHES, the Chinese American Eye Study; H, PM Classification by Hayashi; M, International META-PM Classification; META-PM, meta analyses of pathologic myopia; P, International Photographic Classification and Grading System of Myopic Maculopathy; SEED, the Singapore Epidemiology of Eye Disease; V, PM Classification by Vongphanit.

selected studies, sum totals of these subgroup analyses data may not be equal to that of individuals in the 12 studies.

\section{Quality assessment and publication bias}

Using the 8-item assessment tool to assess the quality of the selected studies, the scores of the studies ranged from 5 to 8 , with an average score of 6.3. Four studies were considered high quality, ${ }^{701419}$ and the remaining studies were moderate quality. ${ }^{612} 1315-1820$ The most common limitation of the selected studies was the lack of a clear description of non-responders.

The funnel plot of 12 selected studies (13 datasets) is shown in the online supplementary figure 1 . After removing each study sequentially for sensitivity analysis, the pooled prevalence of remaining studies did not change significantly compared with the initial results. We adopted the result of the Begg tests and considered that there was no publication bias for the prevalence of MMD.

\section{Prevalence of high myopia and MMD in the world population}

The prevalence of high myopia ranged from $1.3 \%$ to $8.0 \%$, whereas the prevalence of MMD ranged from $0.2 \%$ to $10.7 \%$ given in table 2 . The pooled prevalence of high myopia was $3.0 \%$ (95\% CI: $2.1 \%$ to $4.2 \%$ ) as shown in the online supplementary figure 2 and the pooled prevalence of MMD was 2.1\% (95\% CI: $1.3 \%$ to $3.3 \%$ ). A relevant forest plot was shown in the online supplementary figure 3 . When it comes to MMD within high myopic subjects, the prevalence rate was $47.4 \%$ (95\% CI: $24.3 \%$ to $71.7 \%$ ) (online supplementary figure 4). As for sublesions of MMD, the pooled prevalence of staphyloma was $0.9 \%$ (95\% CI : $0.6 \%$ to $1.4 \%$ ), being the most frequent pathological lesions of MMD. Chorioretinal atrophy at the posterior pole was also common, with a prevalence of $0.6 \%$ (95\% CI : $0.1 \%$ to $2.3 \%)$. Other pathological lesions of MMD such as lacquer cracks and Fuchs' spots were infrequent with prevalence rates of $0.3 \%$ (95\% CI: $0.2 \%$ to $0.4 \%$ ) and $0.2 \%$ (95\% CI: $0.1 \%$ to $0.2 \%$ ), respectively. MMD prevalence within high myopic subjects ranged from $25.3 \%$ to $71.4 \%$. The age-specific and age-sex-specific prevalence of MMD is given in figure 2.

\section{Subgroup analysis of the pooled prevalence of MMD}

The pooled prevalence of MMD among different subgroups is shown in table 3. Online supplementary figures 5-12 show the forest plots of MMD prevalence by several subgrouping methods. In the subgroup of gender, the prevalence of MMD in women is higher than men with statistical significance $(2.6 \%$, $95 \%$ CI $(1.6 \%$ to $4.3 \%)$ for women vs $1.9 \%$, 95\% CI $(1.1 \%$ to $3.5 \%$ ) for men, $\mathrm{p}<0.001)$.

As for the comparison between populations living in different areas, our results show that the prevalence of MMD in rural areas is significantly lower than that in urban districts. Meanwhile, people are at higher risk of having MMD in American and Australia than in Asia.

As for the subgroup analysis of axial length, the prevalence of MMD in subjects with an axial length longer than $26 \mathrm{~mm}$ is higher than subjects with an axial length shorter than $26 \mathrm{~mm}$. Myopes with severer spherical equivalent (SE) refraction levels are more likely to suffer from MMD $(p=0.08)$.

For the subgroup analysis of age group, the prevalence of MMD increased from $1.3 \%(95 \% \mathrm{CI}, 1.0 \%$ to $1.7 \%)$ in the $40-49$ age group to $4.5 \%$ (95\% CI, $2.6 \%$ to $7.8 \%$ ) in the $70+$ age group $(\mathrm{p}<0.001)$. When comparing the prevalence of MMD in earlier studies versus later studies (1993-2006 vs 2007-2019), a time trend was found with the prevalence of MMD being higher in the later study group than earlier study group $(1.3 \%, 95 \% \mathrm{CI}$ $(0.8 \%$ to $2.2 \%)$ vs $3.5 \%, 95 \%$ CI $(1.9 \%$ to $6.3 \%))$.

Different grading schemes also influenced the prevalence rate of MMD. The prevalence of studies using PM Classification by Vongphanit is much lower than that of International META-PM Classification $(1.6 \%, 95 \% \mathrm{CI}(1.0 \%$ to $2.6 \%)$ vs $4.7 \%, 95 \% \mathrm{CI}$ (2.4\% to $8.9 \%))$.

In exploratory meta-regression analyses, significance was found between the prevalence rate of MMD and the following factors: response rate $($ slope $=-1.170, \mathrm{p}<0.0001)$, study quality score $($ slope $=-0.222, \mathrm{p}<0.0001)$, conducted year (slope $=0.068, \mathrm{p}<0.0001)$, male to female ratio (slope $=-2.256$, $\mathrm{p}<0.0001$ ), and myopia degree (slope $=-0.715, \mathrm{p}<0.0001)$.

\section{DISCUSSION}

In the current study, 12 studies with 13 datasets conducted in different parts of the world (China, Taiwan China, Japan, India, 


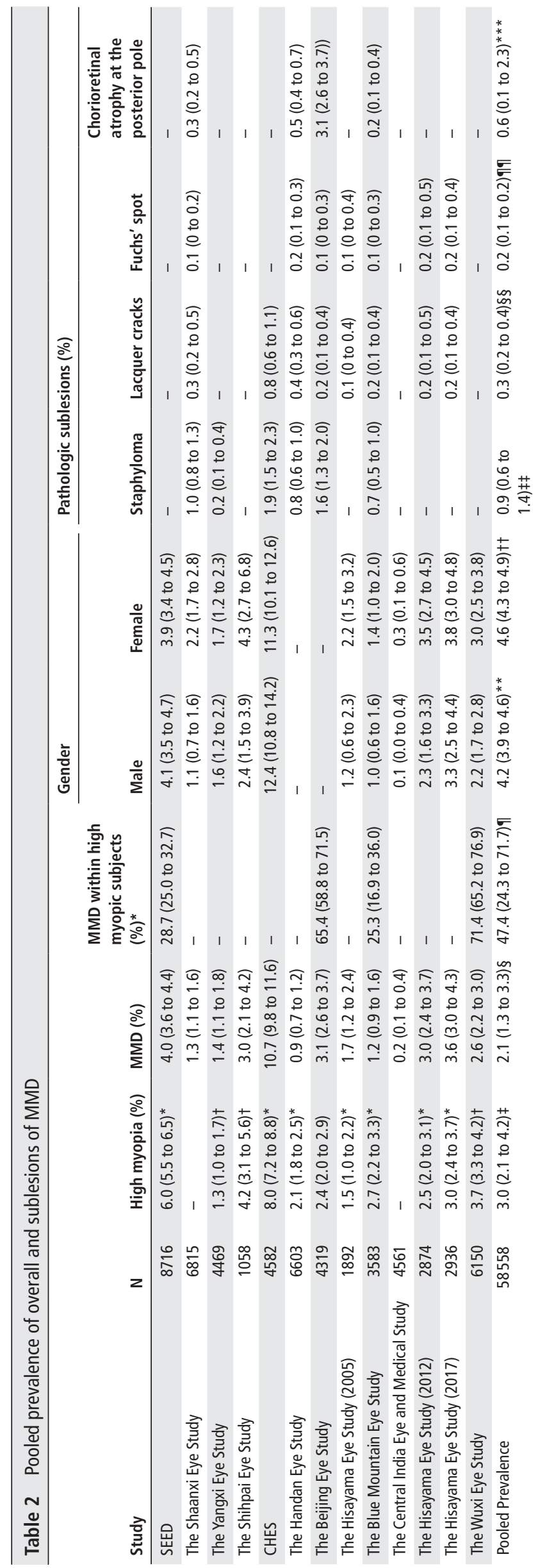


A

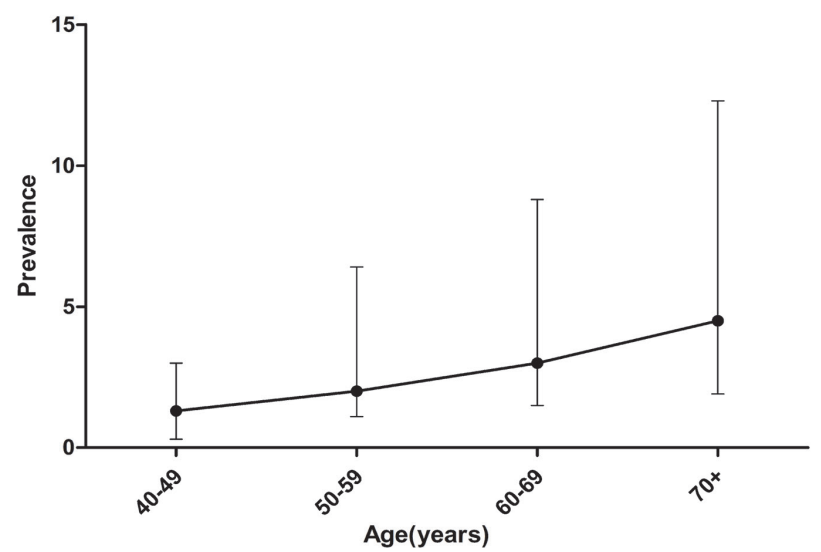

B

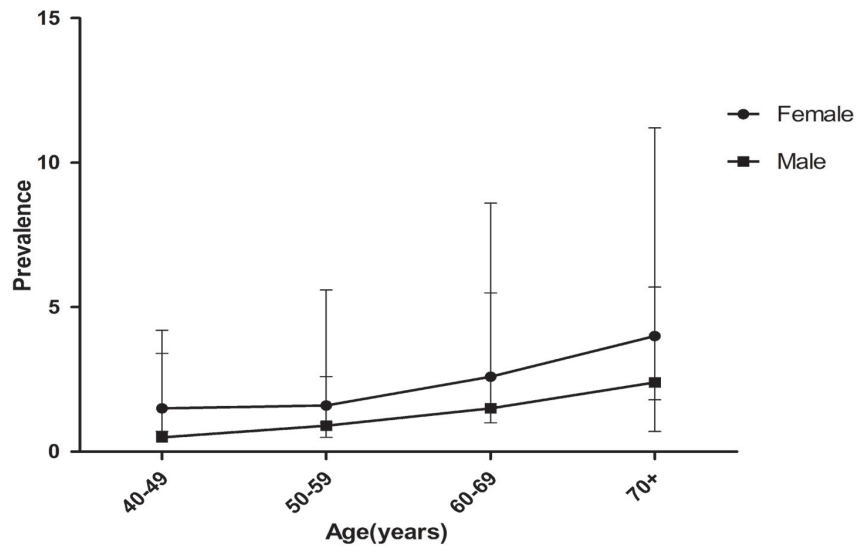

Figure 2 Age-specific and age-sex-specific prevalence of MMD among the world population. (A) Age-specific prevalence of MMD. (B) Age-sexspecific prevalence of MMD. MMD, myopic macular degeneration.

Singapore, Australia and USA) were included, and the pooled prevalence of MMD in this meta-analysis was 2.1\% (95\% CI: $1.3 \%$ to $3.3 \%)$. Most of the included studies were from Asia. Though no meta-analysis of MMD has been published prior, the result is consistent with the traditional view that MMD is a frequently occurring ocular disease in the elderly population and the risk of developing MMD among high myopic subjects is much higher $(47.4 \%, 95 \% \mathrm{CI}: 24.3 \%$ to $71.7 \%)$. In the sublesions of MMD, staphyloma and choroidal atrophy at the posterior pole ranked the first and second most frequent pathological changes in MMD.

In the subgroup analysis of gender, the result indicates that MMD is more common in women than men, in accordance with most of the original investigations. ${ }^{61012-14} 16-20 \mathrm{~A}$ possible explanation lies in the anatomical differences between sexes and discrepancy in career choices between men and women which could have an impact on the pathogenesis of MMD. 131417

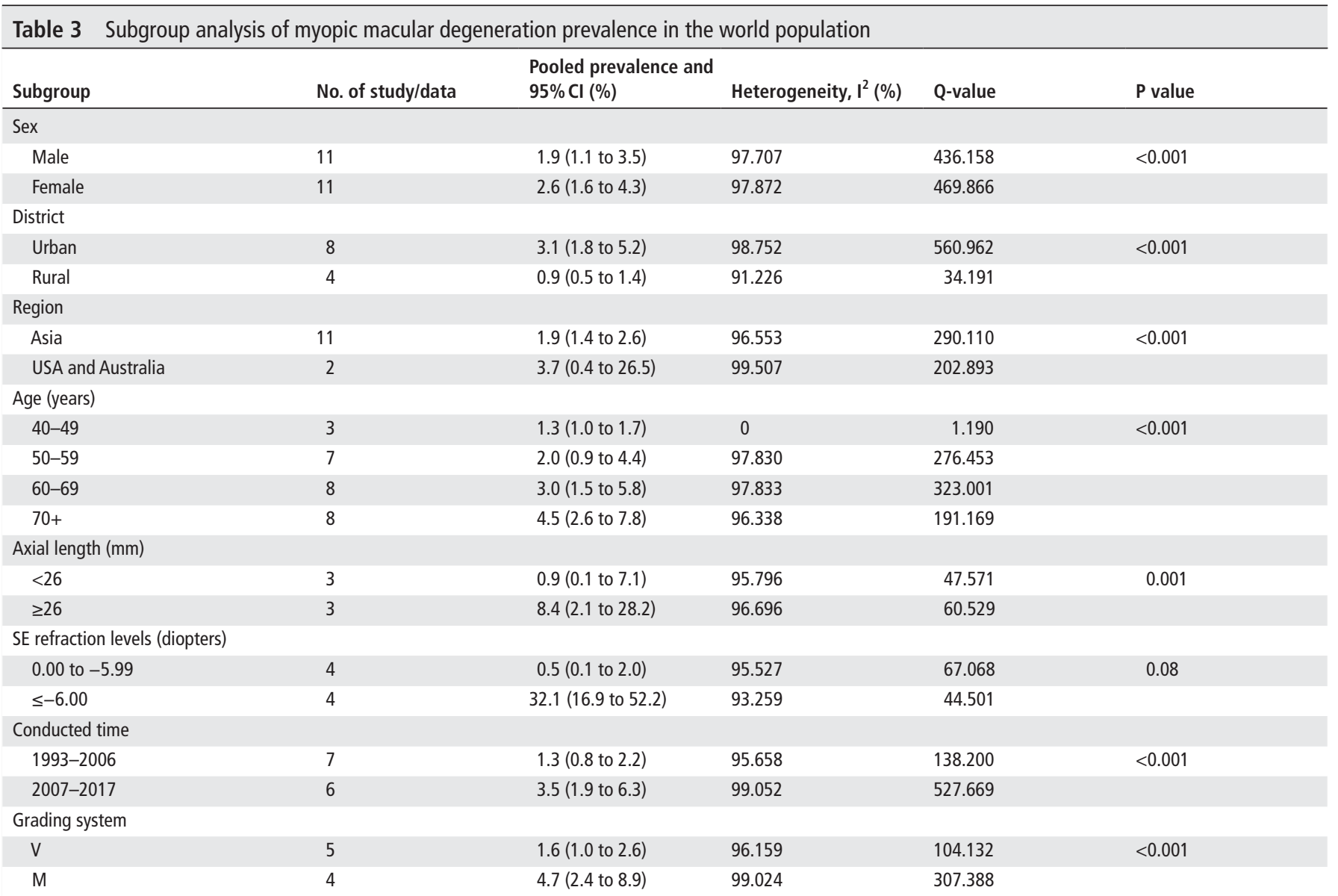

M, International META-PM Classification; META-PM, meta analyses of pathologic myopia; SE, spherical equivalent; $\mathrm{V}$, PM Classification by Vongphanit. 
Specific living areas may contribute to the development of MMD, as results of this meta-analysis show that people living in cities are at higher risk of developing MMD than those in rural districts. Urban citizens tend to have a higher level of education, which is shown to have a strong correlation to more myopes and higher SE refraction levels, thus leading to the severity of MMD. ${ }^{26}$ Residents of Asia have a lower rate of MMD in comparison with those who dwell outside Asia. Lifestyle is considered as an important factor as genetic characteristics may be similar between Chinese Americans and Chinese born and raised locally. The axial length of different races could also contribute to the disparity, which will be discussed further. As there are only two included articles which studied the American and Australian population, the result should be considered with prudence.

In our study, populations without myopia or with a low SE refraction level (0.00 to -5.99 diopters) had a prevalence of only $0.5 \%$ (95\% CI: $0.1 \%$ to $2 \%$ ), whereas the prevalence of people with high myopia ( $\leq-6.00$ diopters) was $>32 \%$, which is consistent with previous studies where it was found that severe refraction error is related to high prevalence of MMD. ${ }^{27}{ }^{28}$ This meta-analysis also found, with significant statistical significance, that the longer the axial length was, the more likely for men to develop MMD. Axial length could affect the thickness of choroid and posterior sclera, which is an important risk factor of a series of complications like MMD. ${ }^{2629-31}$ Possible mechanism could be the varying axial length in different races. ${ }^{32} 33$

The significant difference in the prevalence of MMD in four specific age groups should be highlighted. In the age-specific subgroup analysis, the prevalence of MMD in the $70+$ age group is more than three times that of the 40-49 age group. It has been proven by past research that increasing maculopathy severity has a strong association with older age. ${ }^{32} 33$

Analysis between studies conducted from 1993 to 2006 and from 2007 to 2019 shows that people in the latter subgroup tend to develop MMD in a higher frequency. ${ }^{712} 151820$ Changes in lifestyle could be an important factor in the rise of prevalence. Other changes, such as the improvement of diagnostic methods or advances in ophthalmic devices, could also influence the epidemiology of MMD.

Studies using International META-PM Classification ${ }^{7} 1520$ are found to have a higher MMD prevalence than those using PM Classification by Vongphanit. ${ }^{6} 10121316$ The most possible explanation for this disparity may be the difference in the definition of chorioretinal atrophy as the diagnostic criteria in PM Classification by Vongphanit ${ }^{10}$ is stricter than that in the International META-PM Classification ${ }^{7}$ as the definition of chorioretinal atrophy by Vongphanit also required the copresence of additional myopia-related signs which could assist in differentiating myopic chorioretinal atrophy from the atrophic signs of laser scars, age-related maculopathy or toxoplasmosis, whereas diffuse chorioretinal atrophy, patchy chorioretinal atrophy and macular atrophy were all considered to have MMD in META-PM Classification.

The strength of this meta-analysis lies in the large pooled sample size from wide geographical distribution. In addition, it is one of the few meta-analyses concerning the prevalence of MMD being published, which is needed for a better understanding of the present situation and more advanced clinical guidance. Moreover, the quality assessment of all included studies with clearly defined evaluation tools ensures the quality of this meta-analysis.

However, several limitations should be considered. First, although similar definitions of MMD were used in different studies, inconsistencies in the definition could affect the results to a certain extent. Second, some relevant information in subgroup analysis, such as axial length levels and SE refraction levels, was not available in all selected articles, which may influence the analysis process. Third, residents living in mainland China make up a large proportion of the total study population and could have an impact on the analysis result. In addition to Asia, only studies from the USA and Australia could be found. Consequently, the prevalence of MMD in regions such as Latin America, Africa and Europe would need to be explored for a deeper understanding of the disease.

In conclusion, this meta-analysis offers a comprehensive and up-to-date estimate of MMD among wide populations, with the subgroups of age, gender, district, region, axial length and SE refraction levels analysed. The results of this meta-analysis indicate that the prevalence of MMD remains high and with the discrepancy in different subgroups. Further studies are needed to explore potentially affected factors such as sex, lifestyle, different racial axial length and severity of myopia on the development of MMD. Because the majority of included studies were conducted in Asia, studies from other continents/ethnicities are needed for comprehensive estimates of the prevalence of MMD globally.

Contributors GJ and DZ: designed the study, initiated the collaborative project, revised the paper. MZ, SW and ZL: monitored data collection, wrote the statistical analysis plan and drafted the paper. AC, CAY and YZ: cleaned and analysed the data. GJ and DZ: administrative, technical or logistic support.

Funding This work was supported by National Natural Science Foundation of China $(81873673,81900841)$ and the Fundamental Research Funds of the State Key Laboratory of Ophthalmology.

Competing interests None declared.

Patient consent for publication Not required.

Provenance and peer review Not commissioned; externally peer reviewed.

Data availability statement Data are available upon reasonable request. Data are available upon reasonable request to email guangming27050103@126.com.

\section{ORCID IDs}

Minjie Zou http://orcid.org/0000-0001-7706-6663

Guangming Jin http://orcid.org/0000-0001-9994-6338

\section{REFERENCES}

1 Wong TY, Ferreira A, Hughes R, et al. Epidemiology and disease burden of pathologic myopia and myopic choroidal neovascularization: an evidence-based systematic review. Am J Ophthalmol 2014;157:9-25.

2 Holden BA, Fricke TR, Wilson DA, et al. Global prevalence of myopia and high myopia and temporal trends from 2000 through 2050. Ophthalmology 2016;123:1036-42.

3 Holden BA, Jong M, Davis S, et al. Nearly 1 billion myopes at risk of myopiarelated sight-threatening conditions by 2050 - time to act now. Clin Exp Optom 2015:98:491-3.

4 Lichtwitz O, Boissonnot M, Mercié M, et al. Prevalence of macular complications associated with high myopia by multimodal imaging. J Fr Ophtalmo/ 2016;39:355-63.

5 Chan W-M, Ohji M, Lai TYY, et al. Choroidal neovascularisation in pathological myopia: an update in management. Br J Ophthalmol 2005;89:1522-8.

6 Liu HH, Xu L, Wang YX, et al. Prevalence and progression of myopic retinopathy in Chinese adults: the Beijing eye study. Ophthalmology 2010;117:1763-8.

7 Wong Y-L, Sabanayagam C, Ding Y, et al. Prevalence, risk factors, and impact of myopic macular degeneration on visual impairment and functioning among adults in Singapore. Invest Ophthalmo/ Vis Sci 2018;59:4603-13.

8 Liang YB, Friedman DS, Wong TY, et al. Prevalence and causes of low vision and blindness in a rural Chinese adult population: the Handan eye study. Ophthalmology 2008;115:1965-72.

9 Hsu W-M, Cheng C-Y, Liu J-H, et al. Prevalence and causes of visual impairment in an elderly Chinese population in Taiwan: the Shihpai eye study. Ophthalmology 2004;111:62-9.

10 Vongphanit J, Mitchell P, Wang JJ. Prevalence and progression of myopic retinopathy in an older population. Ophthalmology 2002;109:704-11.

11 Xu L, Wang Y, Li Y, et al. Causes of blindness and visual impairment in urban and rural areas in Beijing. Ophthalmology 2006;113:1134.e1-11. 
12 Luan L. Investigate the prevalence of high myopia and high mopia associated with chorioretinopathy among adults aged 50 or above in Wuxi City. Nanjing Medical Univesity, 2013.

13 Xiao-Ling Z, Ren B-C, Yang J-G. Epidemiology of high myopia retinopathy in rural population in Shaanxi Province. Int J Ophthalmol 2007;7:1464-9.

14 Chen S-J, Cheng C-Y, Li A-F, et al. Prevalence and associated risk factors of myopic maculopathy in elderly Chinese: the Shihpai eye study. Invest Ophthalmol Vis Sci 2012;53:4868-73.

15 Choudhury F, Meuer SM, Klein R, et al. Prevalence and characteristics of myopic degeneration in an adult Chinese American population: the Chinese American eye study. Am J Ophthalmol 2018;187:34-42.

16 Gao LQ, Liu W, Liang YB, et al. Prevalence and characteristics of myopic retinopathy in a rural Chinese adult population: the Handan eye study. Arch Ophthalmol 2011;129:1199-204.

17 Asakuma T, Yasuda M, Ninomiya T, et al. Prevalence and risk factors for myopic retinopathy in a Japanese population: the Hisayama study. Ophthalmology 2012;119:1760-5.

18 Li Z, Liu R, Jin G, et al. Prevalence and risk factors of myopic maculopathy in rural southern China: the Yangxi eye study. Br J Ophthalmol 2019;103:1797-1802.

19 Jonas JB, Nangia V, Gupta R, et al. Prevalence of myopic retinopathy in rural central India. Acta Ophthalmol 2017;95:e399-404.

20 Ueda E, Yasuda M, Fujiwara K, et al. Trends in the prevalence of myopia and myopic maculopathy in a Japanese population: the Hisayama study. Invest Ophthalmol Vis SCi 2019;60:2781-6.

21 Jin G, Zou M, Chen A, et al. Prevalence of age-related macular degeneration in Chinese populations worldwide: a systematic review and meta-analysis. Clin Exp Ophthalmol 2019;47:1019-27.

22 Yang $C$, Zhang L, Zhu P, et al. The prevalence of tic disorders for children in China: a systematic review and meta-analysis. Medicine 2016;95:e4354.
23 Loney PL, Chambers LW, Bennett KJ, et al. Critical appraisal of the health research literature: prevalence or incidence of a health problem. Chronic Dis Can 1998;19:170-6.

24 Boyle MH. Guidelines for evaluating prevalence studies. Evid Based Ment Health 1998:1:37-9.

25 Li H, Mitchell P, Rochtchina E, et al. Retinal vessel caliber and myopic retinopathy: the blue mountains eye study. Ophthalmic Epidemiol 2011;18:275-80.

26 Pan C-W, Zheng Y-F, Anuar AR, et al. Prevalence of refractive errors in a multiethnic Asian population: the Singapore epidemiology of eye disease study. Invest Ophthalmol Vis Sci 2013;54:2590-8.

27 Pan C-W, Dirani M, Cheng C-Y, et al. The age-specific prevalence of myopia in Asia: a meta-analysis. Optom Vis Sci 2015;92:258-66.

28 Saw SM, Katz J, Schein OD, et al. Epidemiology of myopia. Epidemiol Rev 1996;18:175-87.

29 Jonas JB, Holbach L, Panda-Jonas S. Histologic differences between primary high myopia and secondary high myopia due to congenital glaucoma. Acta Ophthalmol 2016;94:147-53.

30 Ikuno Y, Tano Y. Retinal and choroidal biometry in highly myopic eyes with spectral-domain optical coherence tomography. Invest Ophthalmol Vis Sci 2009;50:3876-80

31 Park H-YL, Shin H-Y, Park CK. Imaging the posterior segment of the eye using sweptsource optical coherence tomography in myopic glaucoma eyes: comparison with enhanced-depth imaging. Am J Ophthalmol 2014;157:550-7.

32 Saka N, Ohno-Matsui K, Shimada N, et al. Long-term changes in axial length in adult eyes with pathologic myopia. Am J Ophthalmol 2010;150:562-8.

33 Shih Y-F, Ho T-C, Hsiao CK, et al. Visual outcomes for high myopic patients with or without myopic maculopathy: a 10 year follow up study. Br J Ophthalmol 2006;90:546-50 